\title{
DESARROLLO DE UNA HERRAMIENTA PARA EL REGISTRO DE ESTRUCTURAS CEREBRALES 3D UTILIZANDO TÉCNICAS DE PROCESAMIENTO DE IMÁGENES
}

Hernán Felipe García ${ }^{1,2}$, Jose Bestier Padilla ${ }^{2}$ y Ramiro Arango ${ }^{2}$

${ }^{1}$ Grupo de Investigación en Automática, Universidad Tecnológica de Pereira, Colombia, hernan.garcia@utp.edu.co

${ }^{2}$ Grupo de Investigación en desarrollos tecnológicos GIDET, Universidad del Quindío, Colombia

Recibido: 19 de febrero de 2014

Aceptado: 24 de marzo de 2014

Correspondencia del autor:hernan.garcia@utp.edu.co,

\{jbpadilla, ramy\}@uniquindio.edu.co

\section{RESUMEN}

El registro es una de las tareas más importantes dentro del procesamiento de imágenes, consiste en encontrar una transformación geométrica que relacione los puntos de una imagen y otra con el fin de corregir las diferencias de tamaño, posición, orientación o distorsión espacial que pueda presentarse, de manera que sea posible una comparación directa entre ellas que facilite el análisis por parte del especialista, ya sea para el diagnóstico de una enfermedad o para la planeación de una cirugía extereotáxica. En los últimos años se han propuesto técnicas de registro multimodal, especialmente entre imágenes CT (Tomografía computarizada) y MRI (Resonancia magnética), sin embargo, algunos de los problemas que aún se presentan permanecen sin resolver, y por ello son un campo activo de investigación. Debido a los problemas de exactitud que presentan los modelos de registro rígidos y no rígidos unimodales, los cuales aún no alcanzan la precisión necesaria para aplicaciones de neurocirugía y la necesidad de contar con herramientas de neuronavegación para el apoyo en la planeamiento de cirugías derivadas de procesos de neurocirugía, este artículo propone el desarrollo de una herramienta para el registro de estructuras cerebrales $3 d$ utilizando técnicas de procesamiento de imágenes. Los resultados evidencian que una herramienta de registro permite emparejar un determinado atlas cerebral a un registro histológico de un determinado paciente, además que del sistema desarrollado permite su ejecución en línea lo cual beneficia la funcionalidad del sistema en procesos de neurocirugía.

\begin{abstract}
Medical image registration is one of the most important tasks in image guide surgery. It consists of finding a geometrical transformation that relates the points of images in order to correct for differences in size, position, orientation or spatial distortion that can occur, so it is possible a direct comparison between them to facilitate analysis by the specialist, either for diagnosis of a disease or for planning a stereotaxic surgery. In recent years multimodal registration techniques have been proposed, especially among images CT (computed tomography) and MRI (Magnetic Resonance Imaging). However, some problems still remain unsolved, and are therefore an active field of research. Due to problems of accuracy in models of rigid and non-rigid registration, which have not yet reached the precision required for neurosurgical applications, this article proposes the development of a tool for $3 D$ brain registration using image processing techniques. The results show that a registration tool allows matching a given brain histological atlas of a particular patient. Besides, the developed system can be executed online, which benefits system functionality in neurosurgery procedures.
\end{abstract}

Keywords - Magnetic resonance image, computed tomography, brain atlas, image processing. 


\section{Introducción}

La estimulación cerebral profunda es un procedimiento quirúrgico usado para tratar una variedad de síntomas neurológicos invalidantes, más comúnmente los síntomas debilitantes de la enfermedad de Parkinson, tales como el temblor, la rigidez, el agarrotamiento, el movimiento lento y los problemas para caminar. En dicho procedimiento quirúrgico es necesario localizar estructuras anatómicas como el núcleo subtalámico, que difícilmente son visibles mediante las técnicas radiológicas tradicionales. Es por eso que los cirujanos utilizan atlas cerebrales con el fin de facilitar la localización de dichas estructuras. Un atlas cerebral consta de imágenes del cerebro. En ellas, un experto ha identificado las distintas estructuras anatómicas y/o funcionales, etiquetándolas en una serie de cortes con el fin de definir la región cerebral a analizar [1]. A menudo, los cirujanos consultan frecuentemente atlas cerebrales durante la planificación para mejorar la exactitud en las intervenciones. El objetivo final perseguido con los atlas cerebrales es localizar determinadas estructuras en un sujeto diferente al del atlas. Los atlas cerebrales comúnmente usados en dichos procedimientos quirúrgicos, como son el atlas de Schaltenbrand-Wahren y el atlas de Talairach-Tournoux, no son adecuados, si no se adaptan a la anatomía del paciente, es por ello, que es necesario realizar un registro de éstos sobre las imágenes del paciente [2],[3].

En la cirugía de Parkinson, el planeamiento del procedimiento de estimulación cerebral profunda (DBS) es una fase crucial, debido a que se necesita identificar con un alto grado exactitud, las regiones en las cuales se realizará el implante del microelectrodo de registro. Es por esto que se necesita de una metodología que empareje con un alto grado de exactitud, el registro histológico de un determinado paciente, a un atlas cerebral, el cual permita la identificación de las estructuras cerebrales relacionadas con el núcleo subtalámico [4]. El registro de imágenes consiste entonces en encontrar una transformación geométrica entre una imagen de referencia y una imagen objetivo de forma que sea posible una comparación punto a punto entre ellas con el fin de llevarlas hacia un sistema de coordenadas común [5]. Aunque se han propuesto varias técnicas de registro automático para imágenes médicas [5], [6] [7] [8], el grado de emparejamiento no es lo suficientemente alto para aplicaciones de neurocirugía, esto debido a los procesos de transformación de las características propias de la imagen, por lo tanto no pueden obtenerse resultados confiables [9].

Además, es importante realizar el emparejamiento de las estructuras cerebrales con un alto grado de exactitud con el fin de registrar a un determinado paciente un atlas cerebral, en el cual las estructuras cerebrales de la región objetivo (ganglios basales) sean identificables por el especialista en un ambiente 3-dimensional. Es por esto, que el desarrollo de este trabajo propone un método para registrar una estructura 3D a un determinado estudio histológico de imágenes de resonancia magnética (MRI) de un paciente, el cual sirva como apoyo al especialista en un determinado análisis derivado de la cirugía de estimulación cerebral profunda [10-13].

\section{Marco Conceptual}

\section{A. Registro rígido y no rígido}

El registro de imágenes se realiza con el fin de encontrar una correspondencia entre formas idénticas en dos imágenes diferentes, se requiere encontrar una transformación geométrica de una con respecto a la otra. El registro se aplica en imágenes médicas para alinear dos vistas de un mismo objeto, ya que cuando se hace la toma de este tipo de imágenes, se introducen distorsiones al cambiar el punto de vista del objeto. También es utilizado para correlacionar información obtenida de diferentes fuentes de imagen, por ejemplo, combinando información de los tejidos blandos bien discriminados en una resonancia magnética y la localización detallada de los huesos y cartílagos en una tomografía axial computarizada. Las transformaciones más comunes son: rígidas, afines, proyectivas, perspectivas y globales (1). El uso de una u otra está determinado por la diferencia entre las imágenes que van a ser registradas.

La transformación establece la posición en la que los pixeles de la imagen original serán convertidos en una nueva posición en la imagen registrada. Para el caso del registro rígido la transformación se realiza mediante el cálculo de una transformación basada en la distancia euclídea. 


$$
\begin{aligned}
& \hat{p}=R p+t \\
& R=\left[\begin{array}{cc}
\cos \theta & -\sin \theta \\
\sin \theta & \cos \theta
\end{array}\right]
\end{aligned}
$$

Donde $\mathrm{R}$ es una matriz de rotación $\mathrm{y} t=\left[\begin{array}{ll}\mathrm{t}_{\mathrm{x}} & \mathrm{t} \\ \mathrm{y}\end{array}\right]$ es un vector de traslación. $\mathrm{p}^{\wedge}$ y $\mathrm{p}$ son los puntos 2 dimensionales transformados y originales respectivamente, representados en coordenadas no-homogeneas $[\hat{\mathrm{y}} \ddot{\mathrm{x}}$ ]$^{\mathrm{t}} \mathrm{y}[\mathrm{y} \mathrm{x}]^{\mathrm{t}}$ respectivamente.

Para el caso de la transformación no rígida, se aplica un campo de deformación $\varphi$ a la imagen objetivo para lograr el registro. El campo de deformación $\varphi$ está descrito por una función de transformación $\mathrm{J}(\mathrm{g}(\mathrm{x} ; \mu))$ $: \mathrm{V}_{\mathrm{j}} \rightarrow \mathrm{V}_{\mathrm{i}}$, donde $\mathrm{V}_{\mathrm{j}}$ y $\mathrm{V}_{\mathrm{i}}$ son dominios continuos en los cuales J e I están definidos, y $\mu$ es el conjunto de parámetros de transformación a ser encontrados. El problema de registro de imágenes no rigido puede ser formulado como un problema de optimización como se muestra en la ecuación

$$
\widehat{\mu}=\arg \min _{\mu} S_{\alpha}^{\omega}(I(x), J(g(x ; \mu)))(\mathbf{2})
$$

Para alinear la imagen objetivo transformada $\mathrm{J}(\mathrm{g}(\mathrm{x} ; \mu))$ a la imagen de referencia I, se buscan los parámetros de transformación $\mu$ que minimicen la función

$$
S_{\alpha}^{\omega}(I(x), J(g(x ; \mu)))
$$

\section{Materiales y métodos}

\section{B. Bases de Datos}

Para el desarrollo de este trabajo se utilizaron imágenes pertenecientes a los atlas cerebrales SPL-PNL Brain atlas, imágenes de la base de datos de la Universidad Tecnológica de Pereira, Cuyos detalles se explican a continuación.

SPL-PNL Brain Atlas: Es un atlas cerebral desarrollado a partir de imágenes de resonancia magnética $\mathrm{T} 1$ de un paciente sano, contiene 160 imágenes en cada uno de los cortes (axial, sagital y coronal) y reconstrucciones tridimensionales del cerebro al igual que las estructuras etiquetadas [12]

BD-UTP: Es una base de datos recolectada por el Instituto de Epilepsia y Parkinson del Eje Cafetero NEUROCENTRO en conjunto con la Universidad Tecnológica de Pereira. Esta base de datos contiene grabaciones de procedimientos quirúrgicos de pacientes con enfermedad de Párkinson. Estas grabaciones fueron adquiridas por un resonador de 1.5 teslas, el cual contiene registros de imágenes de tomografía axial computarizada (CT), imágenes de resonancia magnética T1 y T2 (MRI). Estos registros contienen un volumen 3D de la información cerebral de cada paciente. Contiene alrededor de 150 imágenes por estudio [15].

\section{Módulo de registro de imágenes MRI}

Para el desarrollo del módulo de registro, se utiliza la librería registro y segmentación de imágenes médicas ITK y la herramienta de visualización IGSTK con el fin de desarrollar una herramienta para la visualización y registro de estructuras cerebrales en un entorno 3-dimensional [18],[19].

En la figura 1, se muestra el esquema del módulo de registro desarrollado, en el cual se calcula una función de transformación que empareje de manera exacta un determinado regisro histológico de referencia (Atlas cerebral) a un registro de un determinado paciente en el cual se desean visualizar algunas estructuras cerebrales como los ganglios basales (tálamo, glóbulo pálido interno y sus respectivos ventrículos).

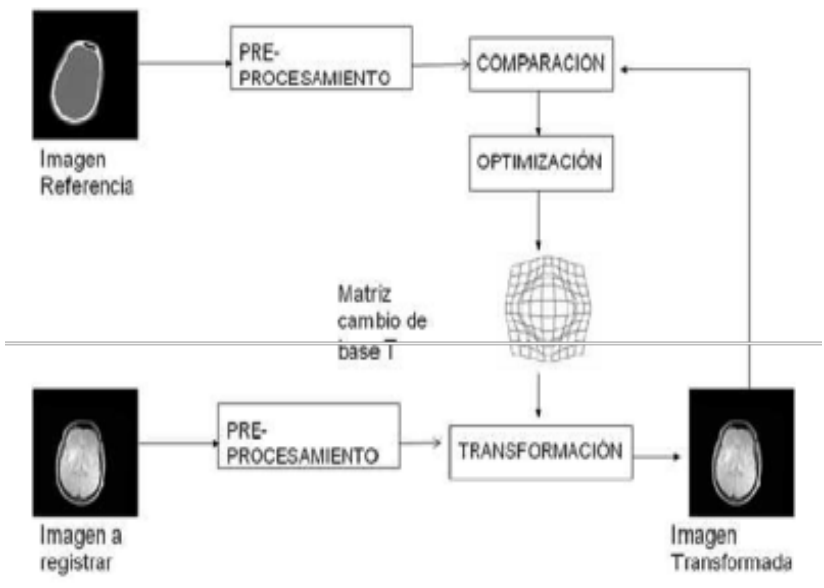

Figura 1. Esquema del algoritmo de registro de imágenes médicas.

D. Herramienta para la visualización y registro de imágenes $3 D$

Para el desarrollo de la herramienta de registro, se utilizó la librería de cirugía guiada por imágenes (IGSTK), la cual es un framework de alto nivel que provee una funcionalidad común para aplicaciones derivadas de cirugía guiada, como lo es la estimulación cerebral profunda [20]. Esta herramienta posee las siguientes 
funcionalidades:

Visualización de entorno 3D

- Carga de registros de imágenes médicas (DICOM)

- Vistas axial, coronal y sagital de los cortes de las imágenes MRI

- Herramienta de transformación de estructuras cerebrales 3-dimensional (traslación, rotación y escala).

- Herramientas para la mejora en la visualización (contraste e iluminación)

\section{Resultados}

\section{E. Resultados para el registro de imágenes MRI}

En lo que respecta a los algoritmos de registro, se aplicaron algoritmos que utilizan transformaciones rígidas para emparejar imágenes CT y MRI a su versión girada respectivamente. Se encontró a través de pruebas de ensayo y error los parámetros de registro que entregaran el menor valor de error entre la imagen de referencia y la imagen objetivo. Se utilizó el optimizador gradiente descendente.

Se debe tener en cuenta que se debe hacer un redimensionamiento de una de las imágenes antes del registro. En la figura 2, se observan los resultados del registro rígido, en los cuales se observa que el grado de emparejamiento de las estructuras registradas es alto y cumple con los parámetros respectivos.
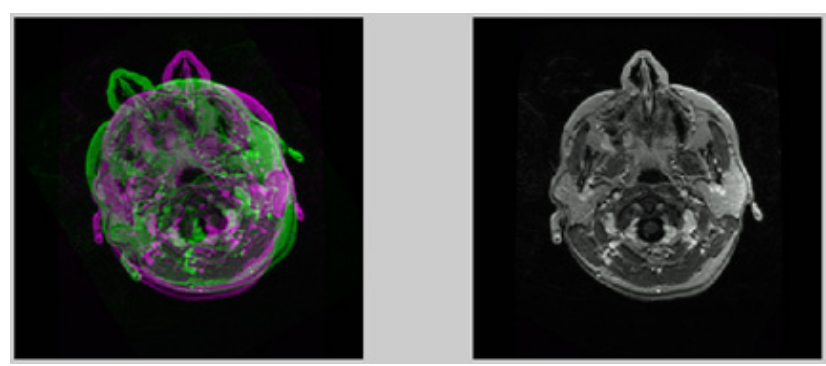

Figura 2. Imágenes MRI antes y después del registro

Además, en la tabla 1 se muestran los valores promedio del error cuadrático medio para diferentes tipos de interpolación de acuerdo al ángulo de rotación; evidenciando un incremento ante la variación en la rotación de las imágenes a registrar.
Tabla 1. Error cuadrático medio luego 30 iteraciones del algoritmo de registro

\begin{tabular}{ccc}
\hline $\begin{array}{l}\text { Angulo } \\
\text { de } \\
\text { rotación }\end{array}$ & $\begin{array}{l}\text { Error } \\
\text { cuadrático } \\
\text { medio (MSE) } \\
\text { Interpolación } \\
\text { Bicúbica }\end{array}$ & $\begin{array}{l}\text { Error } \\
\text { cuadrático } \\
\text { medio (MSE) } \\
\text { Interpolación } \\
\text { Bilineal }\end{array}$ \\
\hline $10^{\circ}$ & 1,9951 & 3,6722 \\
\hline $20^{\circ}$ & 2,2452 & 3,9134 \\
\hline $30^{\circ}$ & 2,2919 & 3,8965 \\
\hline $45^{\circ}$ & 2,3420 & 3,9086 \\
\hline $80^{\circ}$ & 2,0470 & 3,7709 \\
\hline $95^{\circ}$ & 29,060 & 29,992 \\
\hline
\end{tabular}

\section{F. Herramienta de neuronavegación}

En esta sección se muestran los resultados del diseño de la herramienta para el sistema de navegación en una cirugía estereotáxica. Esta herramienta permite la selección de los archivos de RMI del paciente. Se presentan las vistas clásicas: Axial, Sagital, Coronal y los 3 cortes en una escena $3 \mathrm{D}$, con los respectivos cursores, control de Zoom etc. (ver figura 3).

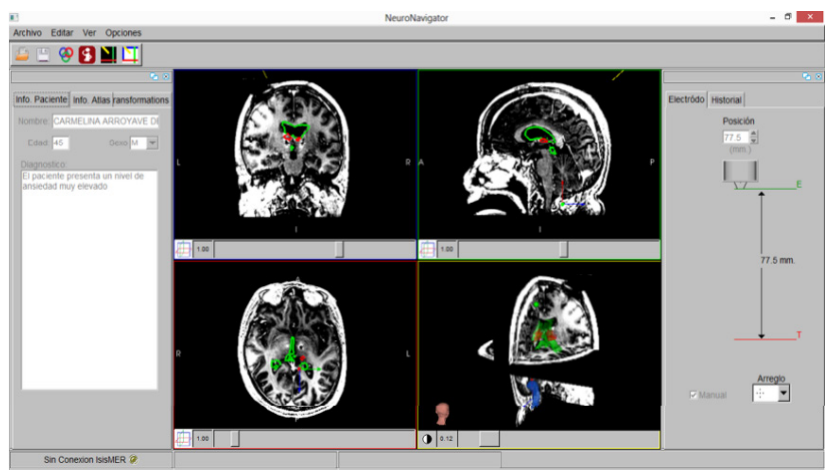

Figura 3. Herramienta de neuronavegación.

Sobre las vistas de RMI se superponen los volúmenes (mesh) de los órganos que componen el Atlas que previamente han sido segmentados o registrados con las RMI (el registrado se hace en otra aplicación que se está construyendo). Este panel tiene las opciones de edición de cada uno de los órganos que conforman el atlas; además su estructura es de árbol con el fin de enlazar todas las estructuras cerebrales que intervienen en el estudio, adicionando opciones como selección, visibilidad y cambio de color (ver figura 4). 


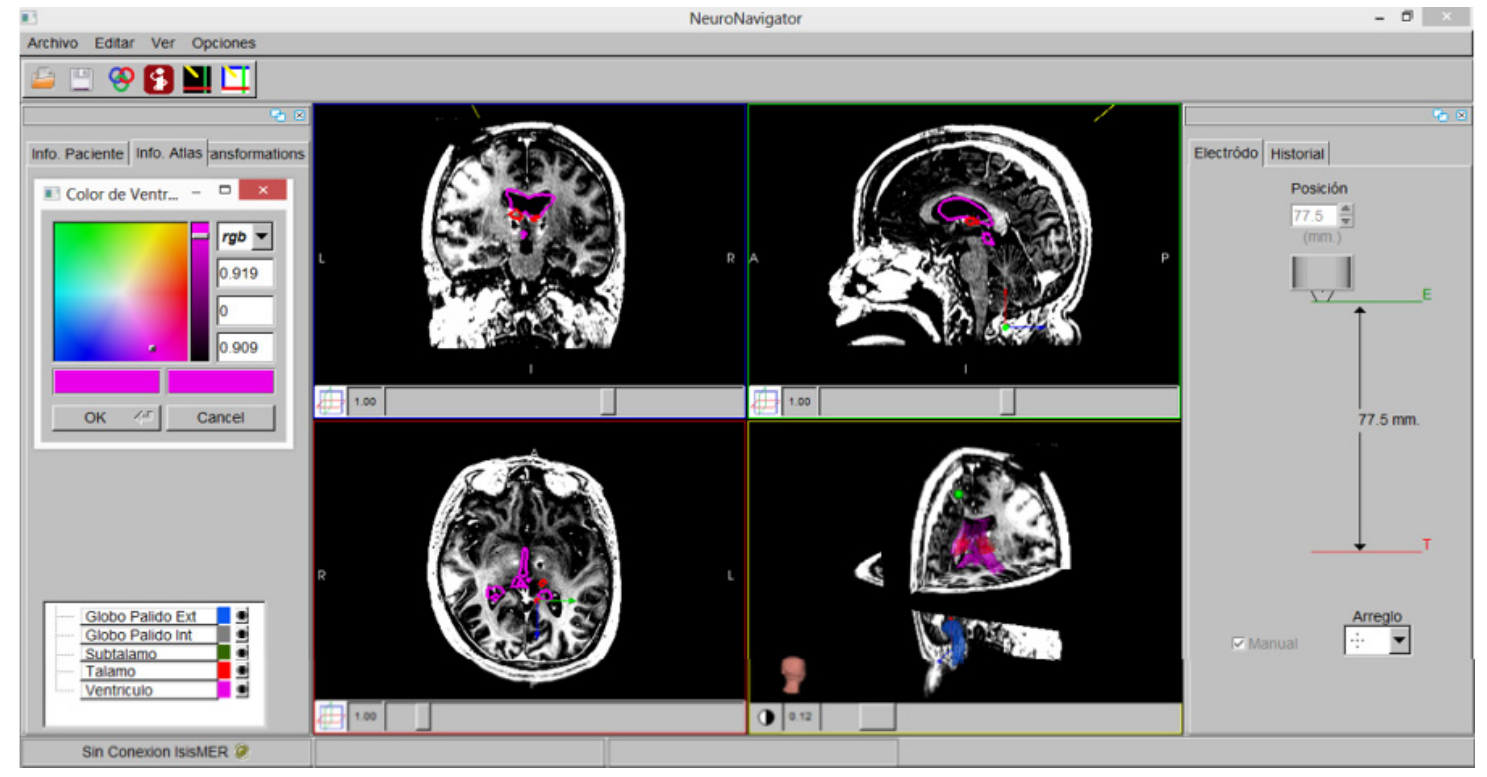

Figura 4. Herramienta de neuronavegación.

El usuario configura los electrodos (puerto IP o serial, etc), y en las imágenes aparece la representación del electrodo. Además al lado aparece una representación de la posición del electrodo que puede ser configurada para desplazarse manualmente (por lo pronto solo esta opción está activada) o siguiendo la señal enviada por el ISIS Mer (en desarrollo) (ver figura 5). El usuario puede activar una vista oblicua en la cual se cambia la vista sagital por un plano paralelo a la dirección de inserción de los electrodos (la idea es que el usuario pueda rotar este plano teniendo como eje el electrodo y que además se cambie otro de las vistas por una vista perpendicular a este plano) (ver figu- ra 6). Otro aspecto importante, es que la aplicación permite la visualización de los cortes entre cada una de las vistas, permitiendo identificar con más claridad opciones como la de la vista oblicua que cuantifique los movimientos de los planos de inserción. Además en el panel izquierdo se observan las opciones para hacer registro sobre las estructuras cerebrales entre las opciones se encuentra:

- Rotación alrededor de los planos Sagital, Axial y Coronal.

- Traslación sobre los planos Sagital, Axial y Coronal.

Fscalado de las estructuras cerehrales

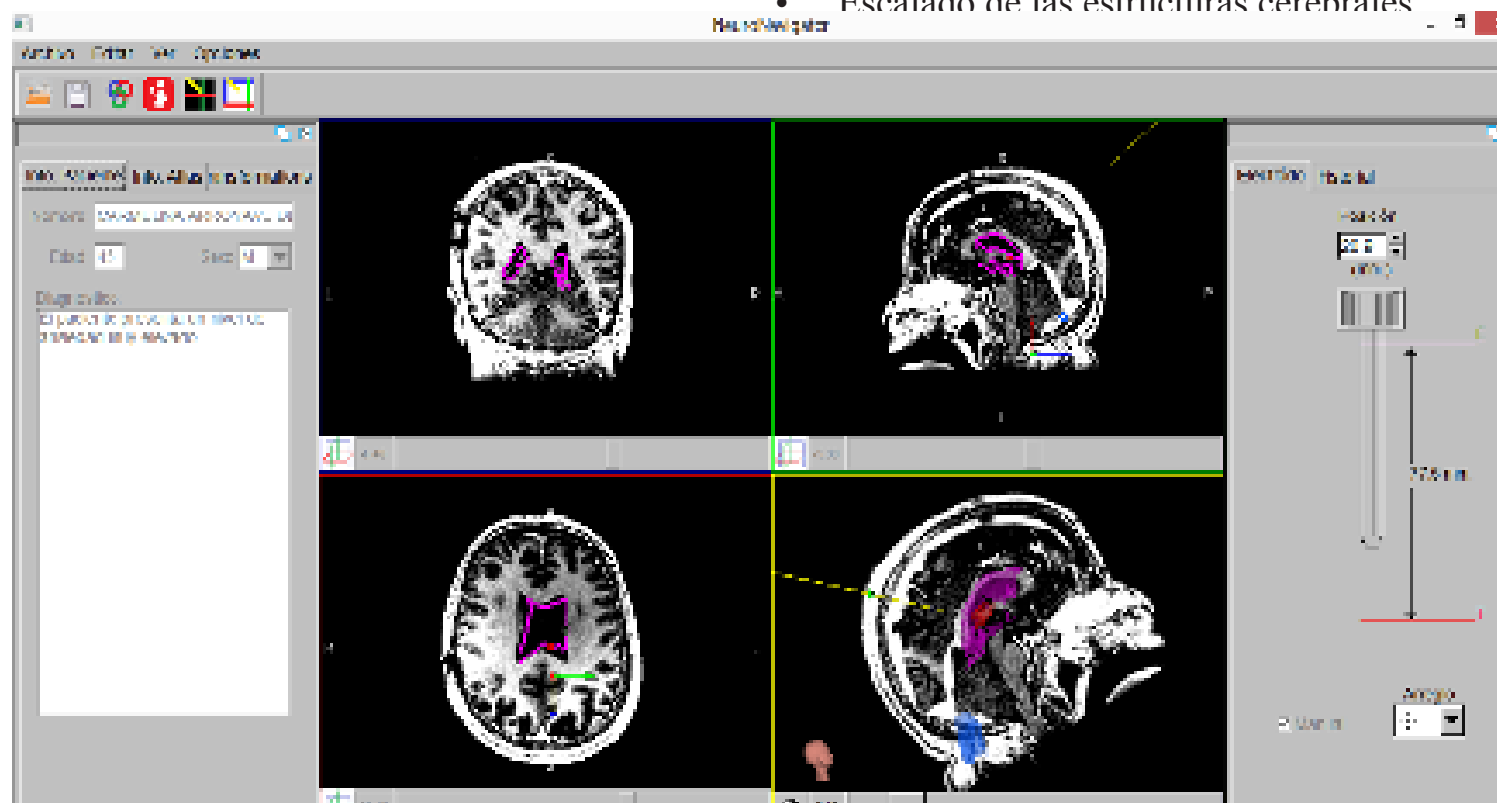

Figura 5. Herramienta de neuronavegación (sistema de tracking del microelectrodo de registro). 


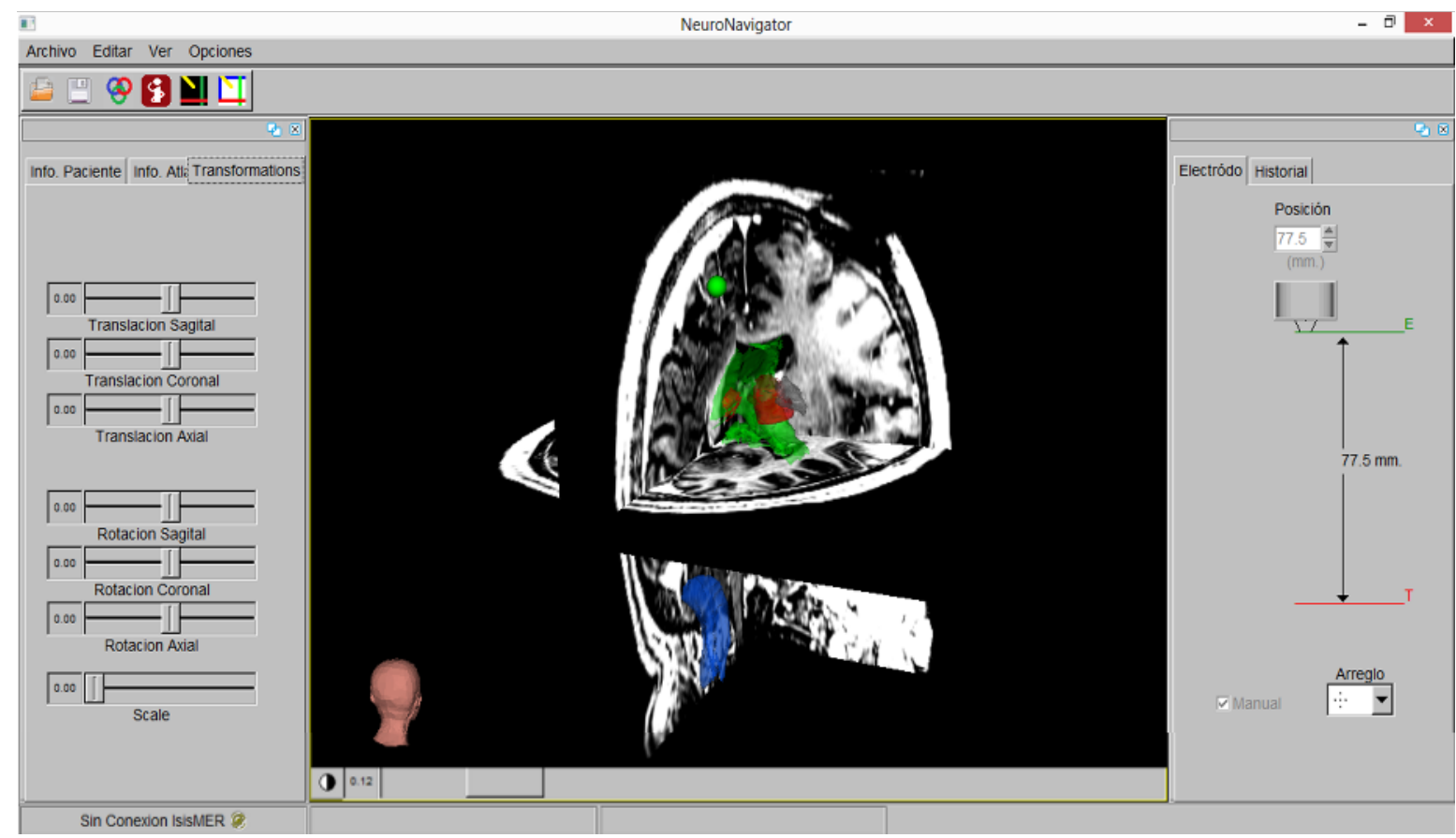

Figura 6. Herramienta de neuronavegación (módulo de transformaciones de estructuras cerebrales).

\section{CONCLUSIONES}

En este trabajo se desarrolló de una herramienta para el registro de estructuras cerebrales 3D utilizando técnicas de procesamiento de imágenes. Los resultados experimentales demuestran que el registro desarrollado en la herramienta, no solo es más robusto ante variaciones de rotación, traslación, escala y algunas deformaciones locales presentes en el estudio médico a registrar. Además este tipo de registro permite una mayor exactitud en el emparejamiento de una determinada estructura cerebral a un registro histológico de referencia (MRI). Así mismo, la herramienta desarrollada brinda un soporte de alto impacto en aplicaciones derivadas de cirugías de neuronavegación, sirviendo de apoyo diagnóstico en el planeamiento de cirugías de estimulación cerebral profunda.

\section{AGRADECIMIENTOS}

Este trabajo se desarrolló gracias a los proyectos "Desarrollo de un sistema efectivo y apropiado de estimación del volumen de tejido activo cerebral para el mejoramiento de los resultados terapéuticos en pacientes con enfermedad de Parkinson intervenidos quirúrgicamente", código 111056934461 y "Desarrollo de un sistema automático de mapeo cerebral y monitoreo intraoperatorio cortical y profundo aplicación a la Neurocirugía", con código 111045426008 , ambos financiados por Colciencias. 


\section{BIBLIOGRAFÍA}

1. van den Elsen, P.A.; Pol, E.-J.D.; Viergever, M.A., "Medical image matching-a review with classification," Engineering in Medicine and Biology Magazine, IEEE , vol.12, no.1, pp.26,39, March 1993. .

2. Fernando Andrés Angarita y Sergio Andrés Acuña. Cáncer de seno: de la epidemiología al tratamiento,2008.

3. Fernando Andrés Angarita y Sergio Andrés Acuña. Presentación inicial de las pacientes con diagnóstico de cáncer de seno en el centro javeriano de oncología, hospital universitario san ignacio, 2010. .

4. Sukhamwang N Muttarak M, Kongmebhol P. Breast calcifications: which are malignant?, 2009. .

5. Mammographic image analysis homepage. .

6. BAZZANI, A, et al. System for automatic detection of clustered microcalcifications in digital mammograms. Italy, 2000. .

7. Marta Lucía Guevara G Damián Alberto Álvarez G. Detección de microcalcificaciones en mamografías digitales, 2006. .

8. iaci.unq.edu.ar/materias/vision/archivos/apuntes/Filtrado\%20Espacial.pdff Universidad Nacional de Quilmes - Ing. en Automatización y Control Industrial Cátedra: Visión Artificial Septiembre de 2005. . 